\title{
CT Volumetric Changes of the Stomach Following Laparoscopic Sleeve Gastrectomy
}

\author{
Ahmed H. Ali, ${ }^{1}$ FRCS, Mohamed El Gharib, ${ }^{2}$ MD.
}

\begin{abstract}
1) Department of General Surgery, Ain Shams University, Cairo, Egypt. 2) Department of Radiology, Ain Shams University, Cairo, Egypt.
\end{abstract}

Background: Laparoscopic sleeve gastrectomy (LSG) has increased in popularity as a definitive bariatric operation. There are no available data in the literature reporting the results regarding the evolution of the size of the gastric tube at middle- or long-term followup correlated to the regain of weight or re-increase of the BMI. Gastric capacity can increase late after sleeve gastrectomy even after performing a narrow gastric tubulization. It is very important to measure objectively the residual gastric volume after sleeve gastrectomy and its increase in order to determine the late clinical results and to indicate the eventual strategy for retreatment. A plain multi-slice abdominal CT with $3 D$ volume rendering image can measure the residual gastric pouch volume, and give details about the anatomy of the residual gastric volume after sleeve gastrectomy.

Patients and methods: From June 2013 till June 2015, 50 morbidly obese patients with a

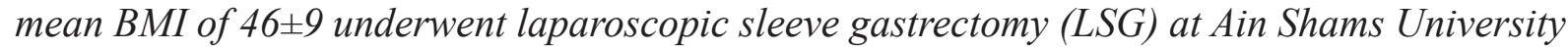
hospitals. A plain multi-slice abdominal CT with $3 D$ volume rendering image was done one and six months after surgery.

Results: 50 obese patients candidate for laparoscopic sleeve gastrectomy were enrolled in our study. There were forty women and ten men with a mean age of 39 years (18-60). Female: male ratio was 80:20. BMI was $46 \pm 9$ (range: $40-58) \mathrm{kg} / \mathrm{m} 2$, with mean body weight 135(105-165) kg. Excess weight loss was achieved after 3, 6, and 12 months after LSG was $23.7 \pm 7,39.9 \pm 14$, and $55.9 \pm 17 \%$, respectively. Multi-slice plain abdominal CT volumetry was done 1 and 6 months postoperative which showed a mean gastric volume of $106 \pm 20 \mathrm{ml}$ one month postoperatively and increased to $167 \pm 20 \mathrm{ml}$ after six months.

Conclusion: A plain multi-slice abdominal CT with $3 D$ volume rendering image to calculate residual gastric pouch after LSG is a noninvasive, relatively accurate method measuring the residual gastric pouch volume after LSG and provides that after 6 months there is no significant correlation between residual gastric pouch volume increase and EBWL.

Key words: Laparoscopic sleeve gastrectomy, obesity surgery, bariatric surgery, multislice CT.

\section{Introduction:}

Obesity is a medical condition in which excess body fat has accumulated to the extent that it may have an adverse effect on health, leading to reduced life expectancy and/or increased health problems. Body mass index (BMI), a measurement which compares weight and height, calculated by dividing the weight by the height of person in meters to the power 2, defines people as overweight (pre-obese) if their BMI is between 25 and $30 \mathrm{~kg} / \mathrm{m}^{2}$, and obese when it is greater than $30 \mathrm{~kg} / \mathrm{m}^{2} .^{1}$

Large-scale American and European studies have found that mortality risk is lowest at a BMI of $20-25 \mathrm{~kg} / \mathrm{m}^{2}$ in nonsmokers and at $24-27 \mathrm{~kg} / \mathrm{m}^{2}$ in current smokers, with risk increasing along with changes in either direction. A BMI above 32 has been associated with a doubled mortality 
rate among women over a 16 -years period. In the United States obesity is estimated to cause an excess 111,909 to 365,000 deaths per year, while 1 million (7.7\%) of deaths in the European Union are attributed to excess weight. On average, obesity reduces life expectancy by six to seven years: a BMI of 30-35 reduces life expectancy by two to four years. While obesity (BMI $>40$ ) also known as Morbid Obesity reduces life expectancy by 10 years. $^{2}$

Until now, bariatric surgery is the only effective treatment for morbid obesity as it leads to sustained weight loss and improvement of comorbidities. ${ }^{3}$

Laparoscopic sleeve gastrectomy (LSG) represents a valid option for morbidly obese patients, either as a primary or as a staged bariatric procedure. Initially, LSG was conceived as a restrictive component of the biliopancreatic diversion and duodenal switch. Later on, LSG has been proposed as a step procedure in high-risk patients, followed by a second step Roux-en-Y gastric bypass or biliopancreatic diversion and duodenal switch. ${ }^{4}$

Recently, LSG has been proposed as a standalone bariatric procedure. Excess weight loss and remission of comorbidities have been reported to take place in a frequency comparable with other well-established procedures. 5

It was observed that some patients who had undergone the laparoscopic vertical sleeve gastrectomy lost significant weight and did not require the second malabsorptive stage. As a consequence, and due to many surgeons' dissatisfaction with the long-term results of laparoscopic adjustable gastric band, laparoscopic vertical sleeve gastrectomy has gained popularity in recent years and is now performed as a stand-alone procedure for weight loss. Since it is relatively recently that large numbers of this operation are being performed, long-term data are lacking. Nevertheless, available data suggest that perioperative and long-term complication rates are about the same as laparoscopic Roux-en-Y gastric bypass, and higher than laparoscopic adjustable gastric band. Weight loss and improvement in obesity-related comorbidities are less than laparoscopic Roux-en-Y gastric bypass but better than laparoscopic adjustable gastric band. ${ }^{6}$

Concerning the diameter of the bougie employed for performing the gastric tube, there is no consensus, but an optimal gastric capacity of 100-120 ml has been suggested. ${ }^{7}$

There are no available data on the literature reporting the results regarding the evolution of the size of the gastric tube at middle- or longterm follow-up correlated to the regain of weight or re-increase of the BMI. Some case reports have demonstrated increase of gastric size and volume of the stomach 3 years after the operation in which a reoperation must be indicated for regain of body weight. ${ }^{8}$

Similar results concerning the percentage of excess weight loss have been reported independently of the bougie size used while performing sleeve gastrectomy. ${ }^{9}$

Gastric capacity can increase late after sleeve gastrectomy even after performing a narrow gastric tubulization. It is very important to measure objectively residual gastric volume after sleeve gastrectomy and its increase in order to determine the late clinical results and to indicate the eventual strategy for retreatment. ${ }^{10}$

A plain multi-slice abdominal CT with $3 \mathrm{D}$ volume rendering image can measure the residual gastric pouch volume and gives details about the anatomy of the residual gastric volume after sleeve gastrectomy.

\section{Patients and methods:}

Patients: From June 2013 till June 2015, 50 morbidly obese patients with a mean BMI of $46 \pm 9$ underwent laparoscopic sleeve gastrectomy (LSG) at Ain Shams University hospitals. Different options for management of morbid obesity were discussed in details with the patients with emphasis on the benefits and more importantly the potential complications and side effects of the different bariatric surgical procedures.

Inclusion criteria: All cases were chosen according to the following criteria:

- BMI more than 40 or more than 35 with an obesity associated co- morbidity 
- Age between 18 - 60 years.

- No endocrinal causes for obesity.

- Psychologically stable.

- Motivation \& acceptance of surgical risks.

Exclusion Criteria:

- Younger than 18 years or older than 60 years.

- Pregnant or breast feeding.

- Suffer from any severe psychiatric illness.

- Significant longstanding heart/lung disease or other severe systemic disease.

- Sweet eaters.

- Patients with significant history of reflux esophagitis.

Clinical evaluation: Clinical evaluation aimed at assessment of degree of obesity, preoperative evaluation and detection of different complications of morbid obesity like hypertension, DM, sleep apnea, skeletal problems, infertility, hernias, history of psychotherapy.

Preoperative investigation:

- Laboratory investigations: $\mathrm{CBC}$, FBS, renal functions, liver functions, coagulation profile and lipid profile.

- Hormonal assay: Thyroid profile to detect any endocrinal causes of obesity.

- Pulmonary evaluation: including X-ray chest \& pulmonary functions if needed.

- Cardiac assessment: ECG \& Echocardiography if needed.

The goals of the preoperative assessment for bariatric surgery were to assess indications and contraindications to bariatric surgery and to treat or optimize medical comorbidities before the surgical treatment. Moreover, another aim was to educate the patients and their families about options and risks of the procedures and to set realistic expectations.

Unless there was a clinical need for early admission, all the patients were to be admitted on the day of the surgery. In uneventful cases we aimed to discharge one day postoperative.

\section{Operative techniques:}

Preoperative Preparation: For prophylaxis against thromboembolic complications, all patients were given low molecular weight heparin. Pneumatic intermittent pressure stocking was used intraoperatively and continued postoperatively till the patient is fully ambulant.

Surgical technique: The patient was put in supine, reverse trendlenburg head up position by 30 degree with legs apart, surgeon stand between patients' leg with assistant to patient's left and camera man on patient's right, five ports were used: one $10 \mathrm{~mm}$ to the left of the umbilicus for camera, one epigastric $5 \mathrm{~mm}$ for self-retaining laparoscopic liver retractor, and two working $12 / 15 \mathrm{~mm}$ at both midclavicluar line for gastrolysis, and stapling, one $5 \mathrm{~mm}$ at left anterior axillary line for the assistant.

Gastrolysis was started from a point 4 $\mathrm{cm}$ from the pylorus up to the angle of His using either ultrasonic shears or a bipolar sealing device (Ligasure). The left crus was completely exposed up to the medial border. A sleeve was created over a $36 \mathrm{~F}$ gastric calibration tube with sequential firings of different color reloads according to stomach thickness excluding all gastric fundus as shown in Figures (1-4).

Methylene blue test done at the end without over sewing the staple line with drain left at gastric bed.

Postoperative follow up: In the postoperative period, all patients were given:

- $3^{\text {rd }}$ generation cephalosporins: in the form of Cefoperazone $1 \mathrm{gm}$ IV on induction of anesthesia then twice per day for one to three days postoperative

- Anticoagulants: Enoxaparin 40.000 IU SC on the night of operation then every 24 hours postoperative till discharge and full ambulation.

- Proton pump inhibitors: Omeprazole $40 \mathrm{mg}$ IV twice per day then by oral administration after discharge for at least a month.

- Antiemetic's: ondancetron $4 \mathrm{mg}$ IV twice to three times per day till discharge then if needed.

In day one or two all patients started oral fluids (if tolerated). All patients were to be discharged 24-72 hours postoperative after meeting the discharge criteria of no bleeding, no leakage and no other complications. 
All patients returned for their first outpatient clinic appointment in twelve days. During the first month, patients were on a liquid only diet that is then advanced to a semi-solid diet for two weeks followed by mashed food for another two weeks. They were then advanced to a regular healthy diet.

CT volumetry: To be arranged one month and six months postoperative to detect the change of the volume of the tube in addition to its correlation with patients weight loss.

Statistical Analysis: Required data were collected tabulated and then statistically analyzed. Analysis of data was done using IBM SPSS software (statistical program for social science version 21). Data analysis was performed by the usual methods of descriptive statistics frequencies and percentages for discrete variables, average, median, and standard deviations for continuous variables. The results were significant (S) with $\mathrm{P}<0.05$ and highly significant (HS) with $\mathrm{P}<0.01$. $\mathrm{P} \geq 0.05$ were regarded non-significant (NS).

\section{Results:}

Enrolled Patients: Between June 2013 and June 2015, 50 obese patients candidate for laparoscopic sleeve gastrectomy were enrolled in this study at Ain Shams University hospitals. There were forty women and ten men with a mean age of 39 (18-60). Female: male ratio was 80:20. BMI was 46 \pm 9 (range: 40-58) $\mathrm{kg} / \mathrm{m}^{2}$, with mean body weight 135(105-165) kg Table (1).

Excess weight loss achieved after 3, 6, and 12 months after LSG was 23.7 $\pm 7,39.9 \pm 14$, and $55.9 \pm 17 \%$, respectively. No cases of partial weight regain were observed during follow-up Figure (5).

Multi-slice plain abdominal CT volumetry was done 1 and 6 months postoperative which showed a mean gastric volume of $106 \pm 20$ $\mathrm{ml}$ one month postoperative and increased to $167 \pm 20 \mathrm{ml}$ after six months Table (2), Figure (6).

\section{Discussion:}

In recent years there has been renewed interest in the surgical treatment of morbid obesity in concomitance with the epidemic of obesity, and application of the laparoscopic techniques to the field of bariatric surgery as well. Bariatric surgery proved effective in providing weight loss of large magnitude, correction of comorbidities and excellent short-term and long-term outcomes. ${ }^{11,12}$

The Laparoscopic Sleeve Gastrectomy (LSG) is being performed more frequently and is currently very "trendy" among laparoscopic surgeons involved in bariatric surgery. LSG is not a new operation as it is the restrictive part of a more complex malabsorpitive bariatric procedure; i.e., the biliopancreatic diversion with duodenal switch (BPD-DS). ${ }^{13}$

At present, LSG is well accepted as a definitive bariatric procedure with low morbidities and acceptable weight loss results. The LSG is a straightforward procedure that can be generally completed laparoscopically, even in the case of an extremely obese patient. It does not involve any digestive anastomosis; no mesenteric defects are created eliminating the risk of internal hernia. ${ }^{14}$

Gastric capacity can increase late after sleeve gastrectomy even after performing a narrow gastric tubulization. It is very important to measure objectively residual gastric volume after sleeve gastrectomy and its increase in order to determine the late clinical results and to indicate the eventual strategy for retreatment. The sleeve size after LSG and its implications are now being controversially discussed. ${ }^{10}$

An initial volume of $100 \mathrm{ml}$ for the remaining stomach has been suggested as adequate. 7 But considerable dilation during follow-up is not an uncommon finding. It is, however, still unclear whether sleeve dilation is a physiological process or represents a cause of insufficient weight loss or secondary weight regain. Dilation is not necessarily linked with weight regain in all patients. ${ }^{10}$ The three-dimensional gastric shape and its possible changes after LSG represent a diagnostic challenge. It can be hypothesized that multislice computed tomography (MSCT) with modern post-processing algorithms might be able to describe more comprehensively and quantitatively the 


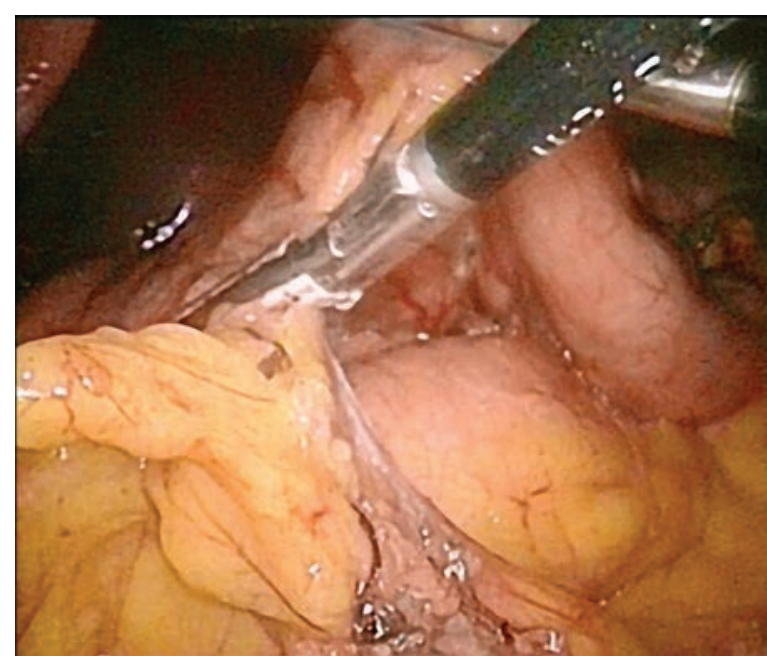

Figure (1): Gastrolysis using Ligasure.

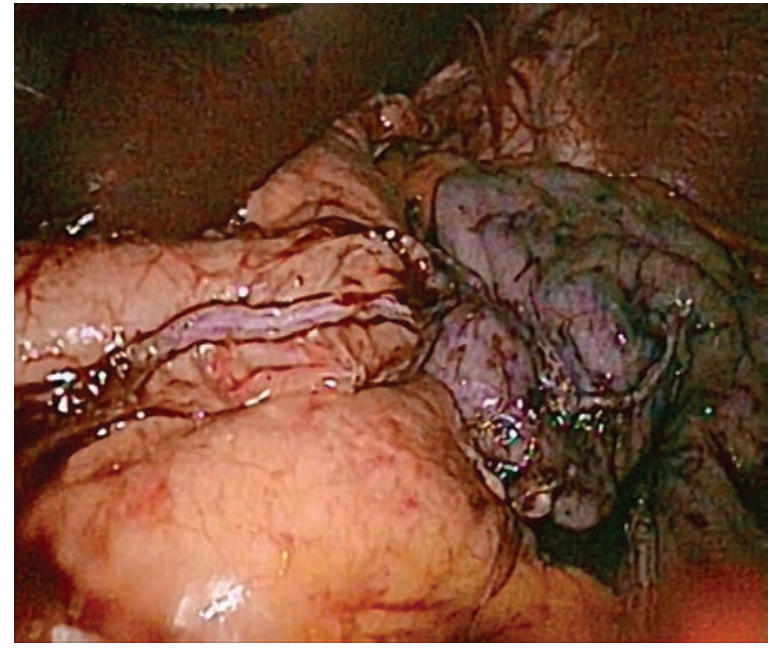

Figure (2): Firing last reload with complete exclusion of the fundus.

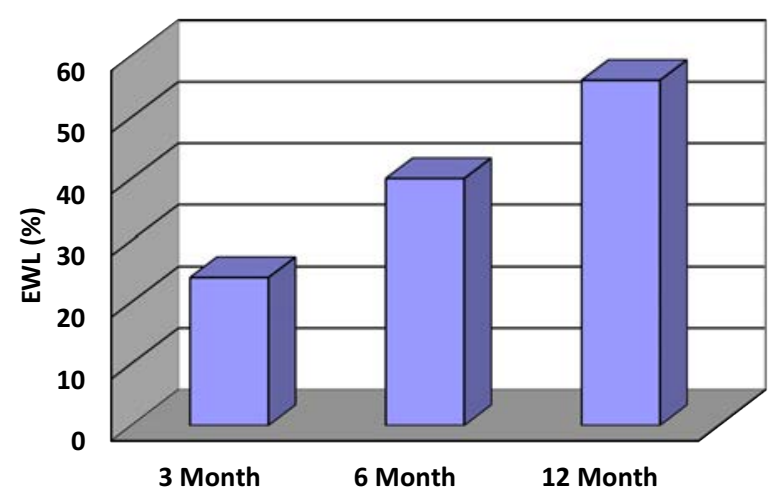

Figure (5): Time [months] weight loss after LSG over time.

anatomical relations after LSG. ${ }^{15}$ Hence, it was the aim of this study to perform a thorough plain multi-slice abdominal CT volumetry with three-dimensional (3D) analysis in patients after LSG and to correlate

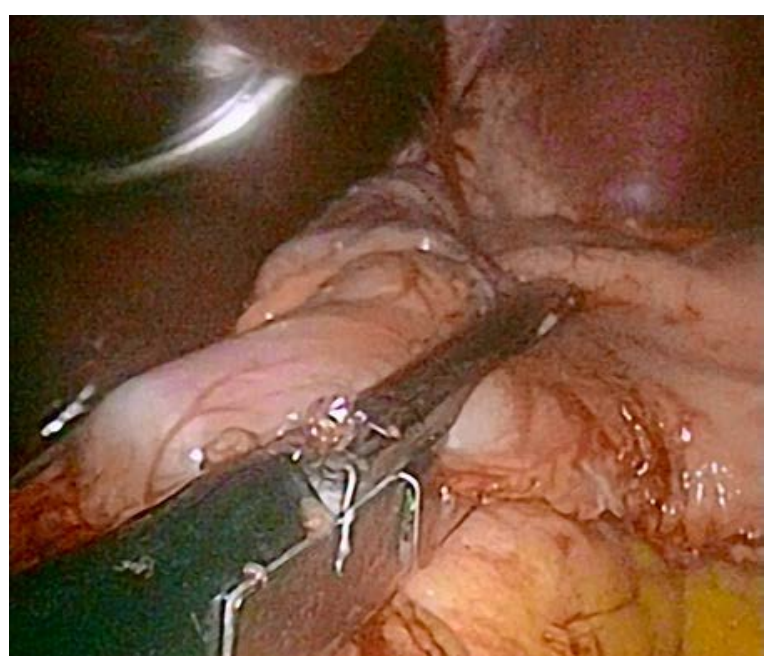

Figure (2): First reload firing.

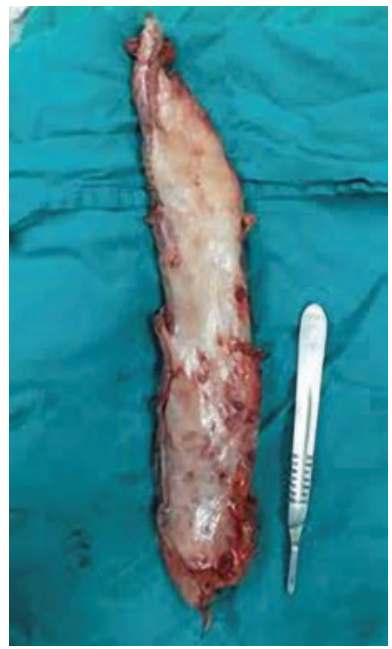

Figure (4): Postoperative specimen for resected stomach.

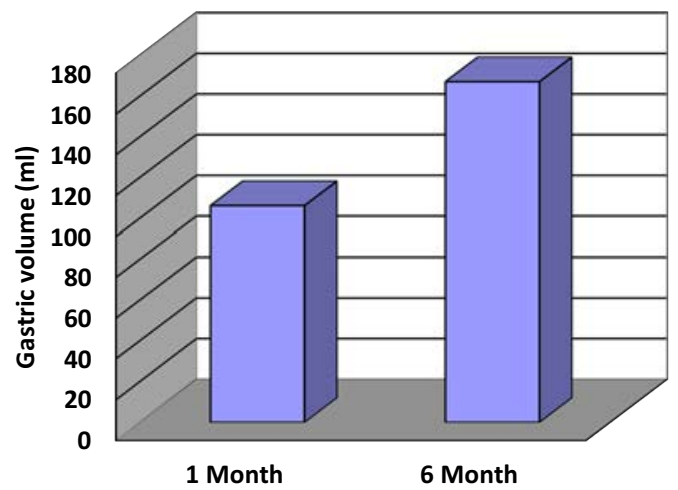

Figure (6): Comparison of sleeve volume change between examinations.

the obtained values with weight loss and the time interval after surgery.

Successful volumetric assessment after LSG by MSCT has been reported by two studies, with a marked dilation of the sleeve 

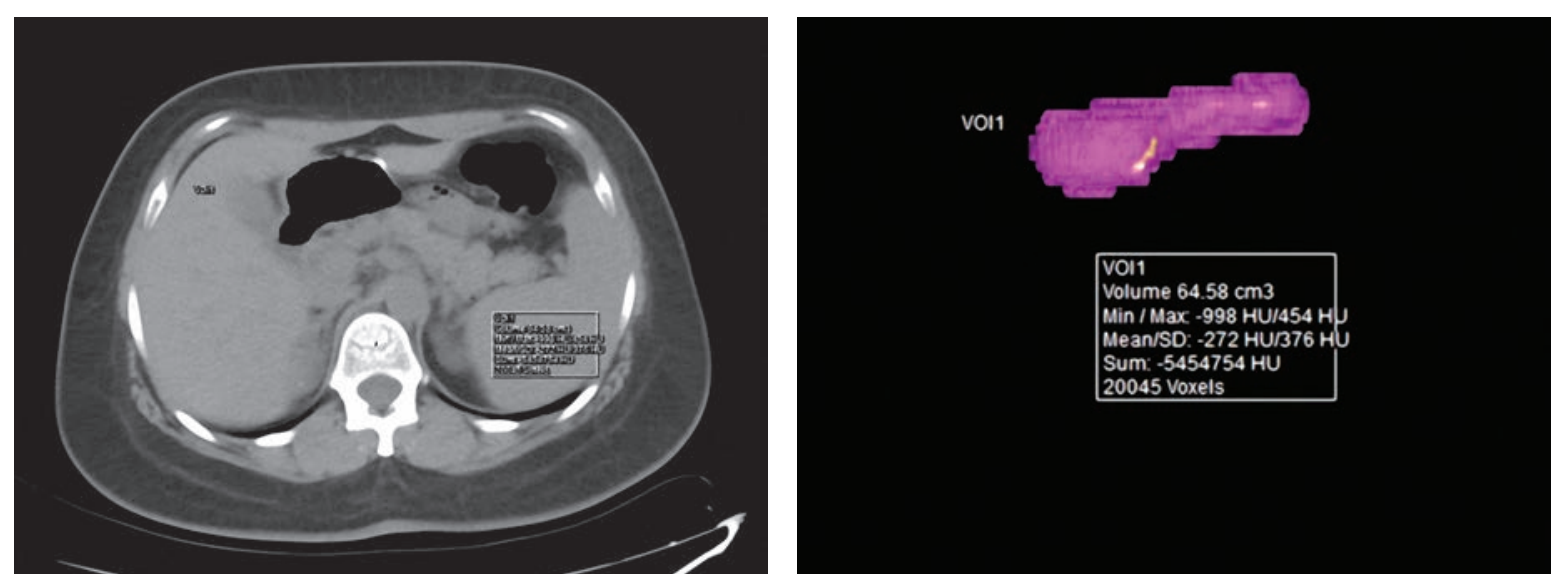

Figure (7): Gastric volume after one month.
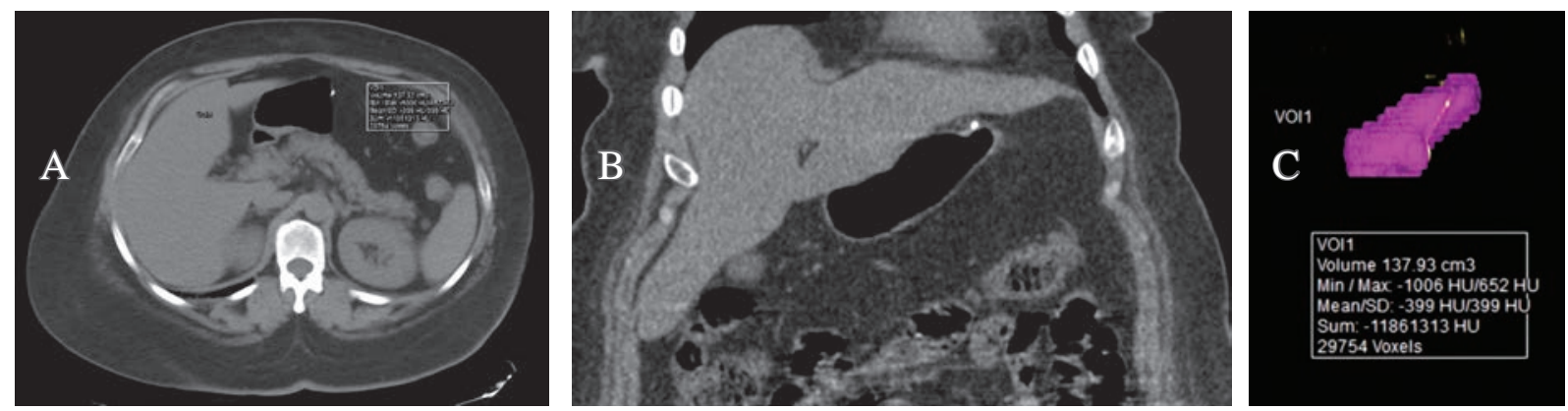

Figure (8): Multislice CT scan of a 34 year old female one month after sleeve gastrectomy (a) axial and (b) coronal reformatted images shows the residual gastric pouch with air inside (c) the $3 D$ volume rendering image showing volume of $137 \mathrm{cc}$.
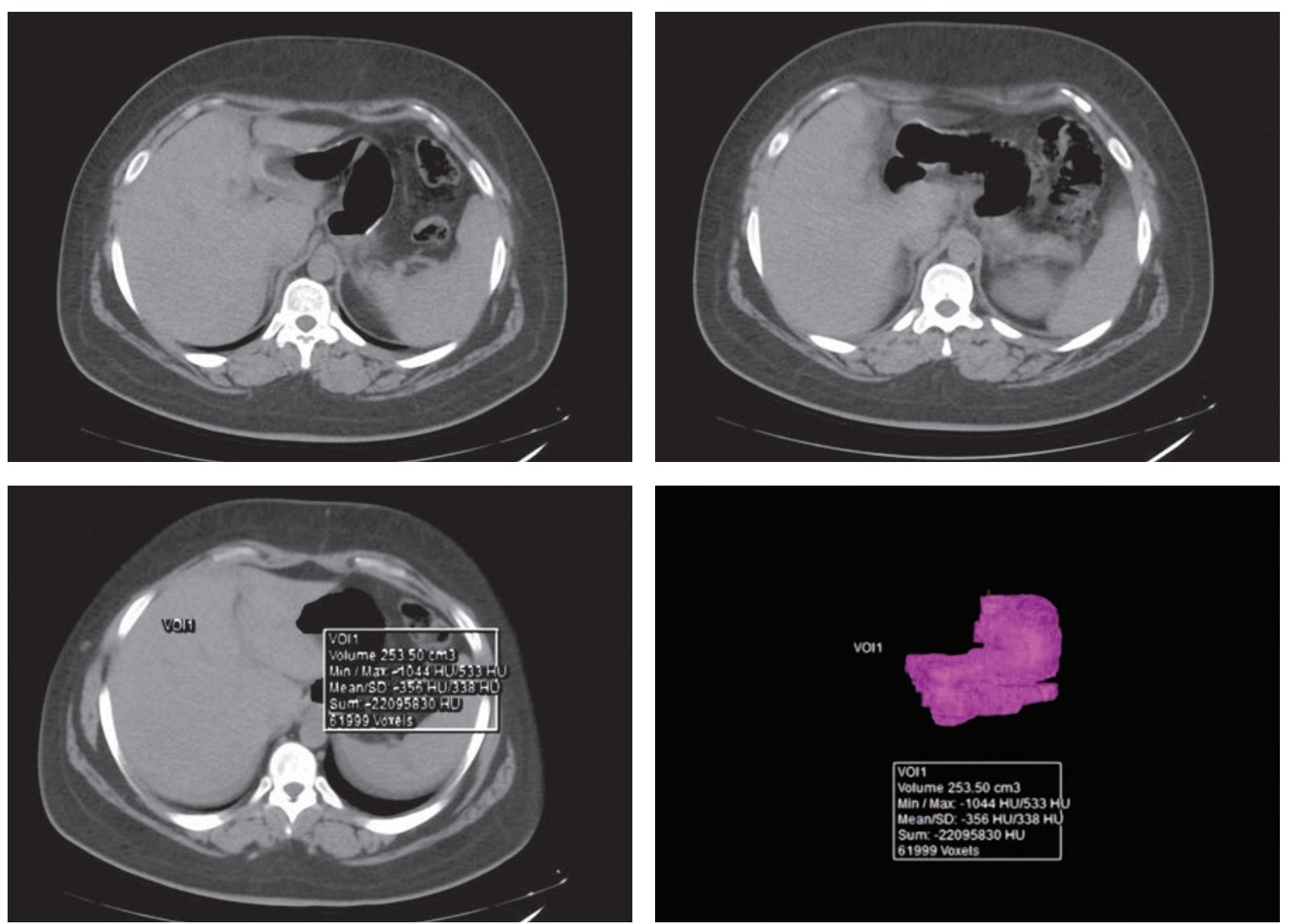

Figure (9): Multislice CT scan of a 38 year old female six month after sleeve gastrectomy reformatted images shows the residual gastric pouch with air inside $(F)$ the $3 D$ volume rendering image showing volume of $253 \mathrm{cc}$. 
Table (1): Patient demographics.

\begin{tabular}{|ll|}
\hline No. of patients & 50 \\
Age (year) & $39($ range 18-60) \\
Sex & \\
$\quad$ Female & $40(80 \%)$ \\
Male & $10(20 \%)$ \\
Preoperative BMI $(\mathrm{kg} / \mathrm{m} 2)$ & $46 \pm 9(40-58)$ \\
Preoperative Weight $(\mathrm{kg})$ & $135(105-165)$ \\
\hline
\end{tabular}

Table 2: CT volumetric study of gastric pouch after LSG Figure (7-9):

\begin{tabular}{|l|l|l|}
\hline Data Finding & After 1 month & After 6 months \\
\hline volume of residual gastric pouch $(\mathrm{ml})$ & $106 \pm 20$ & $167 \pm 20$ \\
\hline
\end{tabular}

being found in an intraindividual comparison of 15 patients during follow-up. ${ }^{10,15}$

Braghetto I. et al., started with a volume of $116 \mathrm{ml}$ early after LSG with dilation to a mean volume of $254 \mathrm{ml}$ after a 2-3-year follow-up.10 Which is comparable to this study, a mean residual gastric pouch volume was $106 \mathrm{ml}$ early after surgery and increased to reach $167 \mathrm{ml} 6$ months postoperative.

The mean residual gastric pouch volume was increased $61 \mathrm{ml}$ (43\% volume increased) while the mean BMI dropped 6 months after surgery from $46 \pm 9$ to $33.9 \pm 14$ with $72 \%$ EWL. This mean that after 6 months there is no significant correlation between residual gastric pouch volume increase and EBWL, and this is comparable to the finding of Parikh M. et al., ${ }^{9}$

Braghetto I. et al., ${ }^{10}$ said that considerable dilation of remaining gastric pouch during follow-up is not an uncommon finding. It is, however, still unclear whether sleeve dilation is a physiological process or represents a cause of insufficient weight loss or secondary weight regain. Dilation is not necessarily linked with weight regain in all patients. However longer follow-up data are, necessary before a connection between secondary sleeve dilation and late weight regain.

\section{Conclusion:}

A plain multi-slice abdominal CT with 3D volume rendering image to calculate residual gastric pouch after LSG is a noninvasive, relatively accurate method measuring the residual gastric pouch volume after LSG and provides that after 6 months there is no significant correlation between residual gastric pouch volume increase and EBWL.

\section{Reference:}

1- Haslam DW, James WP: Obesity. Lancet 2005; 366(9492): 1197-1209.

2- Whitlock G, Lewington S, Sherliker P: Bodymass index and cause-specific mortality in 900000 adults: Collaborative analyses of 57 prospective studies. Lancet March 2009; 373(9669): 1083-1096.

3- Colquitt JL, Picot J, Loveman E, Clegg AJ: Surgery for obesity. Cochrane Database of Systematic Reviews 2009; (2) CD003641.

4- Regan JP, Inabnet WB, Gagner M, Pomp A: Early experience with two-staged laparoscopic Roux-en-Y gastric by-pass as an alternative in the super-super obese. Obesity Surgery 2003; 13(6): 861-864.

5- Dietel M, Crosby RD, Gagner M: The first international consensus summit for sleeve gastrectomy (SG), New York City, October 25-27, 2007; Obesity Surgery 2008; 18(5): 487-496.

6- Shi X, Karmali S, Sharma AM, Birch DW: A review of laparoscopic sleeve gastrectomy for morbid obesity. Obesity Surgery 2010; 20: 1171-1177.

7- Yehoshua RT, Eidelman LA, Stein M, Fichman S, Mazor A, Fichman S, Mazor A, Chen J, Bernstine H, Singer P, Dickman R, Beglaibter N, Shikora SA, Rosenthal RJ, Rubin M: Laparoscopic sleeve gastrectomyvolume and pressure assessment. Obes Surg 2008; 18: 1083-1088.

8- Langer FB, Bohdjalian A, Falbervawer FX, 
Fleischmann E, Reza Hoda MA, Ludvik B, Zacherl J, Jakesz R, Prager G: Does gastric dilatation limit the success of sleeve gastrectomy as a sole operation for morbid obesity? Obes Surg 2006; 16: 166-171.

9- Parikh M, Gagner M, Heacock L: Laparoscopic sleeve gastrectomy: Does bougie size affect mean \%EWL? Short-term outcomes. Surg Obes Relat Dis 2008; 4: 528-533.

10- Braghetto I, Cortes C, Herquiñigo D, Csendes p, Rojas A, Mushle M, Korn O, Valladares $\mathrm{H}$, Csendes A, Maria Burgos A, Papapietro $\mathrm{K}$ : Evaluation of the radiological gastric capacity and evolution of the BMI 2-3 years after sleeve gastrectomy. Obes Surg 2009; 19(9): 1262-1269.

11- Maggard MA, Shugarman LR, Suttorp M, Maglione M, Sugerman HJ, Livingston EH, Nguyen NT, Li Z, Mojica WA, Hilton L:
Meta-analysis: Surgical treatment of obesity. Ann Intern Med 2005; 142: 547-555.

12- Sugerman HJ, Kral JG: Evidence-based medicine reports on obesity surgery: A critique. Int JObes (Lond) 2005; 29: 735-745.

13- Hess DS, Hess DW, Oakley RS: The biliopancreatic diversion with the duodenal switch: Results beyond 10 years. Obes Surg 2005; 15: 408-416.

14- Iannelli A, Facchiano E, Gugenheim J: Internal hernia after laparoscopic Roux-en-Y gastric bypass for morbid obesity. Obes Surg 2006; 16: 1265-1271.

15- Karcz WK, Kuesters S, Marjanovic G, Suesslin D, Kotter E, Thomusch O, Hopt UT, Felmerer G, Langer M, Baumann T: 3D-MSCT gastric pouch volumetry in bariatric surgery-preliminary clinical results. Obes Surg 2009; 19: 508-516. 